\title{
Heart Block in the Athlete - Role of Ion Channel Remodelling as Studied Using a One-dimensional Computational Model of the Atrioventricular Node
}

\author{
Jue Li ${ }^{1}$, Pietro Mesirca ${ }^{2}$, Alicia D’Souza ${ }^{1}$, Yanwen Wang ${ }^{1}$, Shu Nakao ${ }^{1}$, Charlotte Cox ${ }^{1}$, \\ Jules Hancox ${ }^{3}$, Matteo Mangoni ${ }^{2}$, Mark Boyett ${ }^{1}$ \\ ${ }^{1}$ University of Manchester, UK \\ ${ }^{2}$ French National Centre for Scientific Research, France \\ ${ }^{3}$ University of Bristol, UK
}

\begin{abstract}
In swim-trained mice, we previously observed atrioventricular $(A V)$ node dysfunction characterised by first-degree heart block and a prolonged Wenckebach cycle length. Notably, patch clamp recordings from isolated AV node myocytes demonstrated a significant reduction of $I_{f}($ by $\sim 60 \%)$ and $I_{C a, L}($ by $\sim 40 \%$ ) in swimtrained mice as compared to sedentary control mice. The effect of the observed changes in ionic currents was investigated using a one-dimensional computational model of the AV node. This was used in conjunction with a biophysically-detailed model of the rabbit AV node action potential. On decreasing $I_{f}$ by $\sim 60 \%$ and $I_{C a, L}$ by $\sim 40 \%$ to mimic the effect of athletic training, the conduction velocity of the AV node model was reduced by $25 \%$ from 9.5 to $7.1 \mathrm{~cm} / \mathrm{s}$. The reduction was solely the result of the decrease in $I_{C a, L}$. The decrease in $I_{f}$ (modelled in the conventional way as a time-dependent hyperpolarization-activated inward current) had no effect on the conduction velocity, despite experimental evidence that $I_{f}$ can affect $A V$ node conduction. However, if the model of $I_{f}$ was modified to incorporate an instantaneous current, the decrease in $I_{f}$ also contributed to the reduction in the conduction velocity. We conclude that ionic remodelling of the $A V$ node is a key mechanism underlying heart block in the athlete.
\end{abstract}

\section{Introduction}

First- and second-degree heart block, resulting from slower conduction of the action potential through the atrioventricular (AV) node, is common in athletes and has been correlated to the intensity and length of training [1]. In athletes, bradyarrythmias resulting from dysfunction of the cardiac conduction system (including the AV node) can result in syncope [2].

In swim-trained mice, AV node dysfunction characterised by first-degree heart block and a prolonged
Wenckebach cycle length have been observed previously [3]. This was concomitant with diffuse transcriptional, protein and functional remodelling of the hyperpolarization-activated cyclic nucleotide-gated $(\mathrm{HCN})$ and L-type $\mathrm{Ca}^{2+}$ channels known to be involved in AV node action potential generation and conduction [3].

In this study, the effect of the observed changes in ionic currents on $\mathrm{AV}$ node conduction was investigated using a one-dimensional computational model of the AV node. This was used in conjunction with a biophysicallydetailed model of the rabbit AV node action potential [4]. The model of $I_{f}$ in the AV node action potential model was also modified to incorporate an instantaneous current as suggested by Proenza et al. [5].

\section{Methods}

To simulate the AV node action potential, the model of the rabbit AV node action potential (N-type) from Inada et al. [4] was used. General equations are:

$$
\begin{aligned}
& \frac{d V}{d t}=-\frac{I_{\text {total }}}{C_{m}} \\
& I_{\text {total }}=I_{C a, L}+I_{K, r}+I_{f}+I_{s t}+I_{p}+I_{N a C a}+I_{b}
\end{aligned}
$$

where $V(\mathrm{mV})$ is the membrane potential, $t(\mathrm{~ms})$ is the time, $I_{\text {total }}(\mathrm{pA})$ is the total membrane current, and $C_{m}(\mathrm{pF})$ is the membrane capacitance. The total membrane current is the sum of seven ionic currents, which are shown in equation (2).

A one-dimensional (1D) model was used to simulate conduction in the AV node. The model includes 100 elements and each element is $100 \mu \mathrm{m}$ in length. The reaction-diffusion equation was used:

$$
\frac{\partial V}{\partial t}=-\frac{I_{\text {total }}}{C_{m}}+D \frac{\partial^{2} V}{\partial x^{2}}
$$

where $D$ is the diffusion coefficient. $D$ was set to $0.001 \mu \mathrm{S} \mathrm{mm}{ }^{2}$. 
One end of the model (elements 1 to 3 ) was stimulated at $3 \mathrm{~Hz}$. The conduction velocity was calculated from the times of arrival of the action potential at the $41^{\text {th }}$ and $61^{\text {th }}$ elements to avoid the influence of the boundaries.

Patch clamp recordings from isolated AV node myocytes demonstrated a significant reduction of $I_{f}$ (by $\sim 60 \%$ ) and $I_{C a, L}$ (by $\sim 40 \%$ ) in swim-trained mice as compared to sedentary control mice. The measured current-voltage $(I-V)$ relationships of $I_{C a L}$ and $I_{f}$ are shown in Figure 1C,D. To simulate the effect of athletic training, the conductance of $I_{f}$ in the model was reduced by $60 \%$ and the conductance of $I_{C a, L}$ in the model was reduced by $40 \%$. The simulated $I-V$ relationships of $I_{C a, L}$ and $I_{f}$ are shown in Figure 1A,B.

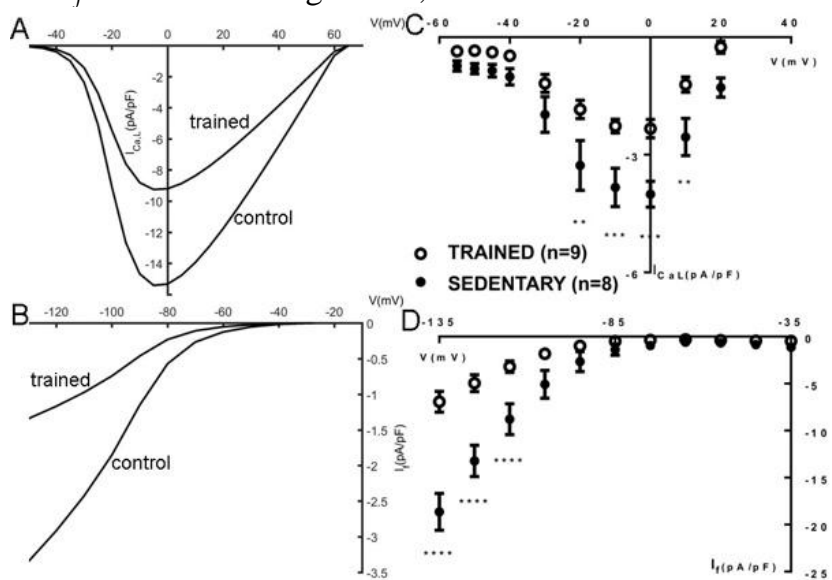

Figure 1. A and B, simulated $I-V$ relationships of $I_{C a, L}(\mathrm{~A})$ and $I_{f}(\mathrm{~B})$ in control and trained AV node cells. C and D, measured $I-V$ relationships of $I_{C a, L}(\mathrm{C})$ and $I_{f}$ (D) in control and trained AV node cells.

\subsection{Simulation of AV node conduction using original AV node ( $\mathrm{N}$ type) model}

The effect of exercise training on AV node conduction was simulated based on the data in Figure 1A,B. Results shows that the conduction velocity of the AV node action potential was reduced by $25 \%$ from 9.5 to $7.1 \mathrm{~cm} / \mathrm{s}$, as shown in Figure 2A,B. Figure 2C shows the time course of $I_{f}$ and $I_{C a, L}$ during the action potential in the control and trained conditions in the computer simulation. The slowing of the conduction velocity was primarily the result of the reduction in $I_{C a, L}$ : decreasing $I_{f}$ alone resulted in a reduction in the conduction velocity of $0.85 \%$ and decreasing $I_{C a, L}$ alone resulted in a reduction in the conduction velocity of $24 \%$. The lack of effect of $I_{f}$ is consistent with our earlier simulations [8], but it is inconsistent with experimental data showing that $I_{f}$ does influence AV node conduction [6-7].

\subsection{Instantaneous component of $I_{f}$}

In the case of $\mathrm{HCN} 2$, there is evidence that there is an instantaneous $\left(I_{\text {inst }}\right)$ component of $I_{f}$ as well as a timedependent component $\left(I_{h}\right)$ of $I_{f}$ from Proenza et al. [5]. Figure 3 (Figure 1C in [5]) shows the correlation between $I_{\text {inst }}$ and $I_{h}$ amplitudes. A Pearson correlation revealed a significant positive covariation between these amplitudes (slope $=0.16, P=0.003, r=0.25$ ). These observations suggested that the same process governs production of $I_{h}$ and $I_{i n s t}$, and that both currents may flow through the same channel [5].
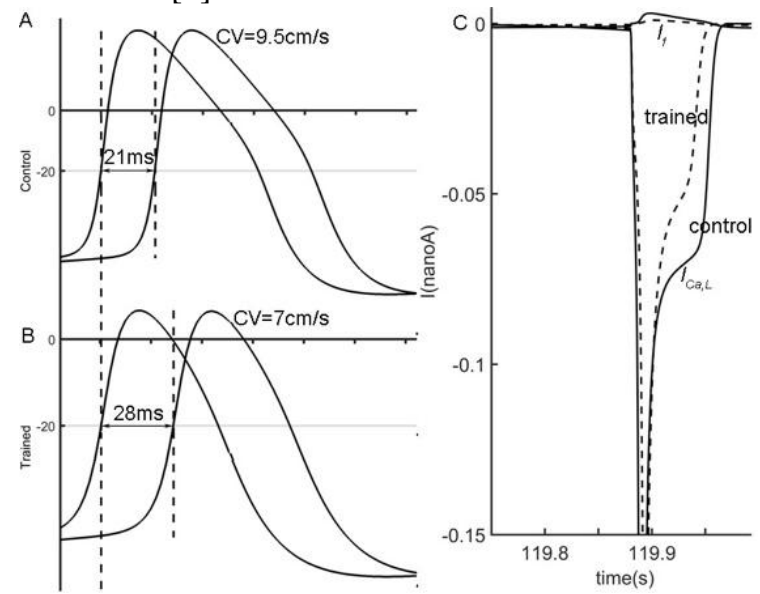

Figure 2. Simulations of effect of athletic training. A: action potential recorded from $41^{\text {th }}$ and $61^{\text {th }}$ elements of the 1D model of the control (A) and trained (B) AV node. C, $I_{f}$ and $I_{C a, L}$ in control (solid line) and trained (dashed line) AV node cells.

I $h(\mathrm{pA} / \mathrm{pF})$

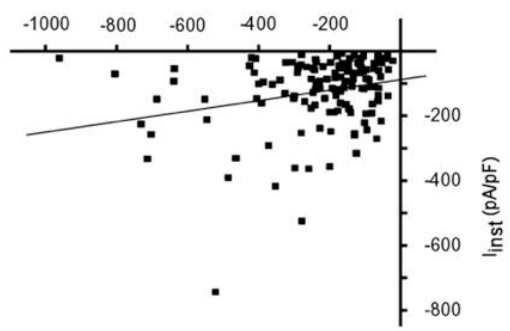

Figure 3. Correlation between $I_{\text {inst }}$ and $I_{h}$ amplitudes. $I_{\text {inst }}$ density is plotted as a function of $I_{h}$ density in the same cells $(n=138)$ in response to voltage steps to $-150 \mathrm{mV}$. The line is a linear fit of the data and indicates a positive covariance $(P=0.003)$ between $I_{\text {inst }}$ and $I_{h}$. From Proenza et al. [5].

The electrophysiological properties of $\mathrm{HCN} 4$ expressed in COS-7 cells were examined by Ishii et al. [9]. Figure 4A shows representative current traces of HCN4 current $\left(I_{f}\right)$ from their study. Figure 4B shows $I-V$ relationships. The closed circles indicate the amplitude of the instantaneous current measured at the beginning of hyperpolarizing pulses. The open circles indicate the amplitude of $I_{f}$ measured at the end of pulses. The similarity of the two $I-V$ relationships suggests both currents flow through the same channel. 
At present, no $I_{f}$ model includes an instantaneous component.

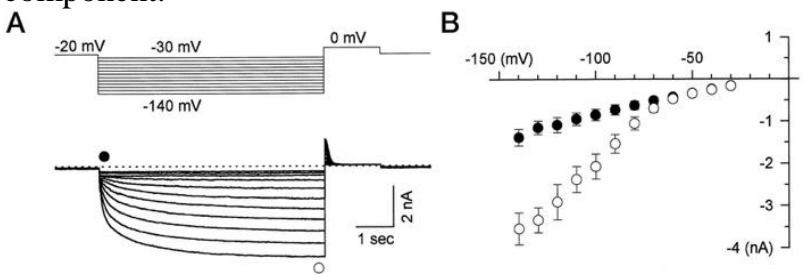

Figure 4. Voltage-dependent gating of HCN4. A, representative current recordings of the HCN4 current $\left(I_{f}\right)$. The initial current $\left(I_{\text {inst }}\right)$ and the steady-state current $\left(I_{f}\right)$ were measured at the times indicated $(\bullet$ and $\circ$, respectively). $B, I-V$ relationships of $I_{\text {inst }}$ and $I_{f}$. From Ishii et al. [9].

\subsection{Modification of $I_{f}$ to incorporate an instantaneous current $\left(I_{\text {inst }}\right)$}

To incorporate an instantaneous current, $I_{\text {inst }}$, three parameters must be determined, the reversal potentials for $I_{\text {inst }}$ and $I_{h}$, the $I-V$ relationship of $I_{\text {inst }}$, and the correlation between $I_{\text {inst }}$ and $I_{h}$.

According to Proenza et al. [5], the reversal potentials for $I_{i n s t}$ and $I_{h}$ do not differ significantly. Hence, the reversal potentials for $I_{i n s t}$ and $I_{h}$ were set to be the same as the reversal potential for $I_{f}$ in Inada's original AV node model $(-30 \mathrm{mV})$. Based on the $I-V$ relationship of $I_{\text {inst }}$ (shown in Figure 4B) measured by Ishii et al. [9], a linear $I-V$ relationship of $I_{\text {inst }}$ was used. According to the results from Proenza et al. [5] (Figure 3) and Ishii et al. [9] (Figure 4B), the ratio of $I_{\text {inst }}$ to $I_{h}$ varies from $\sim 0.3$ to $\sim 1$.

Let $I_{f}=I_{\text {inst }}+I_{h}$.

The model of $I_{h}$ was set as the original model of $I_{f}$, shown in equation (4):

$$
\begin{aligned}
y_{\infty} & =\frac{1}{1+\exp \left(\left(V-V_{1 / 2}\right) / k\right)} \\
\tau_{y} & =0.25+2.0 \exp \left(-(V-(-70))^{2} / 500\right) \\
\frac{d y}{d t} & =\frac{y_{\infty}-y}{\tau_{y}} \\
I_{h} & =g_{h} y\left(V-E_{f}\right)
\end{aligned}
$$

where $y$ is the activation variable of $I_{h}, y_{\infty}$ is the voltage-dependent steady-state value of $y, \tau_{y}$ is the voltage-dependent time constant of $y, V_{l / 2}$ is the voltage at which half activation occurs, and $k$ is a factor determining how steeply the activation curve changes with voltage. $g_{h}$ is conductance of $I_{h}$.

The $I-V$ relationship of $I_{\text {inst }}$ was set to be linear [9]. The model of $I_{\text {inst }}$ is:

$$
I_{\text {inst }}=g_{\text {inst }}\left(V-E_{f}\right)
$$

where $g_{\text {inst }}$ is the conductance of $I_{\text {inst }}$.

To obtain the same action potential shape and the same spontaneous cycle length, on adding an instantaneous component of $I_{f}$, equivalent background current was subtracted.

$$
I_{b}=g_{b}\left(V-E_{b}\right)
$$

where $g_{b}$ is conductance of $I_{b}$ and $E_{b}$ is the reversal potential for $I_{b}$.

The ratio of instantaneous to time-dependent components, $g_{\text {inst }} / g_{h}$, was set to $0.3,0.5$ and 1 to exam the effect of $I_{f}$ on AV node conduction. Table 1 shows modified parameters of $I_{b}$ with $g_{\text {inst }} / g_{h}=0.3,0.5$ and 1 . Figure 5 shows simulated traces of $I_{f}$ incorporating an instantaneous component (from a single AV node cell) with $g_{\text {inst }} / g_{h}=0.3,0.5$ and 1 . The bottom set of traces $\left(g_{\text {inst }} / g_{h}=1\right)$ roughly match the traces shown in Figure 4A.

Table 1 Matching $I_{b}$ to $g_{\text {inst }} / g_{h}$

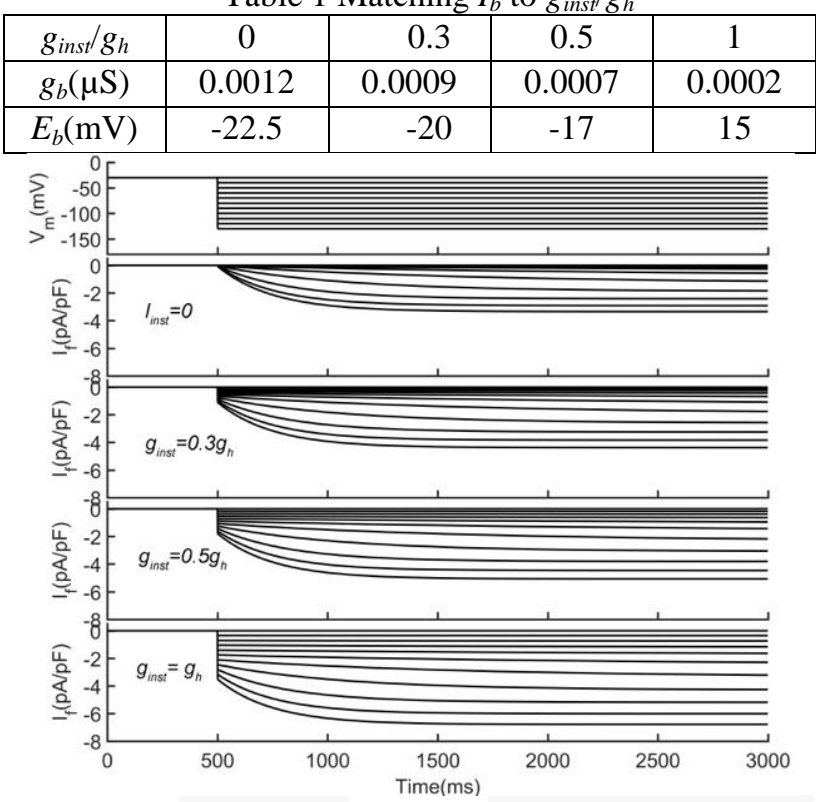

Figure 5. Simulated traces of $I_{f}$ incorporating an instantaneous component (from a single AV node cell). The ratio of instantaneous to time-dependent components, $g_{\text {inst }} / g_{h}=0.3,0.5$ and 1 .

\section{Results}

\section{1. $\quad$ Effect of $I_{f}$ on $\mathrm{AV}$ node conduction and $A V$ node pacemaking}

Incorporating an instantaneous current, $I_{\text {inst }}$ ( $g_{\text {inst }} / g_{h}=0.3,0.5$ and 1 ), significantly increases $I_{f}$ in single cell simulations (Figure 6A). The effect of $I_{f}$ on AV node conduction was calculated using the $1 \mathrm{D}$ model. Figure $6 \mathrm{~B}$ shows that blocking $I_{f}$ decreases $\mathrm{AV}$ node conduction velocity $(\mathrm{CV})$ and the decrease in $\mathrm{CV}$ increases from $0.85 \%$ to $18.5 \%$ as $g_{\text {inst }} / g_{h}$ increases (from 0 to $100 \%$ ).

A single AV node cell model was used to simulate the effect of $I_{f}$ on AV node pacemaking. Liu et al. [10] reported that blocking $I_{f}$ increases the spontaneous cycle 
length of the AV node (by $~ 73.56 \%$ ). Table 2 shows that CL increases as $g_{\text {inst }} / g_{h}$ increases after blocking $I_{f}$. It shows that $g_{\text {inst }} / g_{h}$ should be more than 0.7 to represent the experiment.

Table 2 Effect of the instantaneous component of $I_{f}$ on AV node pacemaking.

\begin{tabular}{|c|c|c|c|}
\hline & $g_{\text {inst }} / g_{h}$ & CL $(\mathrm{ms})$ & Change \\
\hline Control & 0 & 462 & -- \\
\hline \multirow{4}{*}{ No $I_{f}$} & 0 & 471 & $1.88 \%$ \\
\cline { 2 - 4 } & 0.3 & 528 & $14.2 \%$ \\
\cline { 2 - 4 } & 0.5 & 581 & $25.7 \%$ \\
\cline { 2 - 4 } & 0.7 & 671 & $45.2 \%$ \\
\cline { 2 - 4 } & 0.9 & 1023 & $121.4 \%$ \\
\cline { 2 - 4 } & 1 & $\infty$ & $\infty$ \\
\hline
\end{tabular}
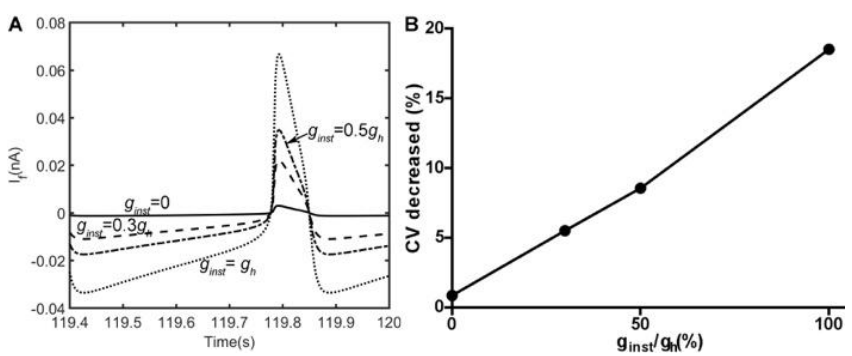

Figure 6. A, traces of $I_{f}$ during the spontaneous AV node action potential when $g_{\text {inst }} / g_{h}=0.3,0.5$ and 1 . B, decrease in AV node conduction velocity on blocking $I_{f}$ after incorporation of an instantaneous component of $I_{f}$.

\subsection{Effect of athletic training on AV node conduction and pacemaker activity}

Table 3 Exercise training is predicted to affect the pacemaker activity of the AV node.

\begin{tabular}{|c|c|c|c|}
\hline & $g_{\text {inst }} / g_{h}$ & CL $(\mathrm{ms})$ & Changes \\
\hline Control & 0 & 462 & -- \\
\hline \multirow{3}{*}{$40 \% I_{f}$} & 0 & 524 & $13.4 \%$ \\
\cline { 2 - 4 }$+{ }^{*}$ & 0.3 & 568 & $22.9 \%$ \\
\cline { 2 - 4 } & 0.5 & 603 & $30.5 \%$ \\
\cline { 2 - 4 } & 0.7 & 646 & $39.8 \%$ \\
\cline { 2 - 4 } & 0.9 & 762 & $64.8 \%$ \\
\cline { 2 - 4 } & 1 & 746 & $61.4 \%$ \\
\hline
\end{tabular}

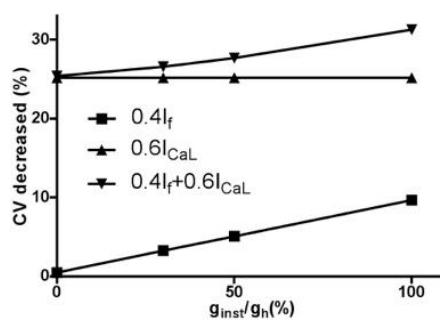

Figure 7. Decrease in AVN conduction velocity caused by exercise training with different patterns of ion channel remodelling.

To simulate the effect of athletic training on AV node conduction and spontaneous cycle length, $0.4 g_{h}$ and 0.6 $g_{C a, L}$ were used. The results shows that the reduction of $I_{f}$ and $I_{C a, L}$ significantly slowed AV conduction (Figure 7) and increased the spontaneous cycle length of the AV node (Table 3 ).

\section{Conclusion}

We conclude that ionic remodelling of the AV node is a key mechanism underlying heart block in the athlete.

\section{Acknowledgements}

This work is supported by British Heart Foundation project grant PG/17/4/32689.

\section{References}

[1] J. Hoogateen et al., "Recommendations and cardiological evaluation of athletes with arrythmias," Netherlands Heart Journal, Vol. 12, pp. 157-164, 2004.

[2] H. Ector et al., "Bradycardia, ventricular pauses, syncope, and sports," Lancet, Vol. 2, pp. 591-594, 1984.

[3] R. L. Verrier et al., "I $I_{f}$ inhibition in the atrioventricular node by ivabradine causes rate-dependent slowing of conduction and reduces ventricular rate during atrial fibrillation," Heart Rhythm, Vol. 11, pp. 2288-2296, 2014.

[4] S. Inada et al., "One-dimensional mathematical model of the atrioventricular node including atrio-nodal, nodal, and nodal-his cells," Biophys J, Vol. 97, pp. 2117-2127, 2009.

[5] C. Proenza, D. Angoli et al., "Pacemaker channels produce an instantaneous current," J. Biol. Chem, Vol. 277, pp. 5101-5109, 2002.

[6] Y. Saeed, I. Temple et al., "Structural and functional remodeling of the atrioventricular node with aging in rats: The role of hyperpolarization-activated cyclic nucleotidegated and ryanodine 2 channels," Heart Rhythm, Vol. 15, no. 5, pp. 752-760, 2018.

[7] M. Baruscotti, A. Bucchi et al., "Deep bradycardia and heart block caused by inducible cardiac-specific knockout of the pacemaker channel gene HCN4," PNAS, Vol. 108, no. 4, pp. 1705-1710, 2011.

[8] J. Li, I. Temple and M. Boyett, "Theoretical study of the role of the funny current $\left(I_{f}\right)$ and the background inward current $\left(I_{b}\right)$ in atrioventricular nodal conduction," Computing in Cardiology, Vol. 41, pp. 865-868, 2014.

[9] T. M. Ishii, M. Takano et al., "Molecular characterization of the hyperpolarization-activated cation channel in rabbit heart sinoatrial node," J Biol Chem, Vol. 274, no. 18, pp. 12835-12839, 1999.

[10] J. Liu, P. J. Noble, et al., "Role of pacemaking current in cardiac nodes: Insights from a comparative study of sinoatrial node and atrioventricular node," Prog Biophys Mol Biol., Vol. 96, pp. 294-304, 2008.

Address for correspondence:

Professor Mark Boyett

Division of Cardiovascular Sciences, University of Manchester CTF Building, 46 Grafton Street, Manchester M13 9NT, UK mark.boyett@manchester.ac.uk 